\title{
The Time-Optimal Problems for Controlled Fuzzy R-Solutions
}

\author{
Andrej V. Plotnikov, Tatyana A. Komleva, Irina V. Molchanyuk \\ Odessa State Academy of Civil Engineering and Architecture, Odessa, Ukraine \\ E-mail:a-plotnikov@ukr.net,t-komleva@ukr.net,i-molchanyuk@ukr.net \\ Received April 21, 2011; revised May 12, 2011; accepted May 15, 2011
}

\begin{abstract}
In the present paper, we show the some properties of the fuzzy R-solution of the control linear fuzzy differential inclusions and research the time-optimal problems for it.
\end{abstract}

Keywords: Fuzzy Differential Inclusions, Control Problems, Time-Optimal Problems, Fuzzy R-Solution

\section{Introduction}

The first research of the differential equations with setvalued right-hand side has been fulfilled by A. Marchaud [1,2] and S. C. Zaremba [3]. In the early sixties, T. Wazewski [4,5], A. F. Filippov [6] had been obtained fundamental results about existence and properties of solutions of the differential equations with set-valued right-hand side (differential inclusions). Connection deriving between differential inclusions and optimum control problems was one of the most important outcomes of these papers. These outcomes became impulse for development of the theory of differential inclusions [7-9].

Considering of the differential inclusions required to study properties of set-valued maps, i.e. an elaboration the whole tool of mathematical analysis for set-valued maps $[7,10,11]$.

In work [12] annotate of an R-solution for differential inclusion is introduced as an absolutely continuous setvalued maps. Various problems for the R-solution theory were considered in $[8,13]$. The basic idea for a development of an equation for R-solutions (integral funnels) is contained in [14].

In the eighties the last century the control theory in the conditions of uncertainty began to be formed. The control differential equations with set of initial conditions [15-17], control set differential equations [18-21] and the control differential inclusions [21-32] are used in the given theory for exposition of dynamic processes.

In recent years, the fuzzy set theory introduced by Zadeh [33] has emerged as an interesting and fascinating branch of pure and applied sciences. The applications of fuzzy set theory can be found in many branches of re- gional, physical, mathematical, differential equations, and engineering sciences. Recently there have been new advances in the theory of fuzzy differential equations [34-47] and inclusions [48-53] as well as in the theory of control fuzzy differential equations [54-57] and inclusions [57-59].

In this article we consider the some properties of the fuzzy R-solution of the control linear fuzzy differential inclusions and research the time-optimal problems for it.

\section{The Fundamental Definitions and Designations}

Let $\operatorname{comp}\left(R^{n}\right)\left(\operatorname{conv}\left(R^{n}\right)\right)$ be a set of all nonempty (convex) compact subsets from the space $R^{n}$,

$$
h(A, B)=\min _{r \geq 0}\left\{S_{r}(A) \supset B, S_{r}(B) \supset A\right\}
$$

be Hausdorff distance between sets $A$ and $B, S_{r}(A)$ is $r$-neighborhood of set $A$.

Let $E^{n}$ be the set of all $u: R^{n} \rightarrow[0,1]$ such that $u$ satisfies the following conditions:

1) $u$ is normal, that is, there exists an $x_{0} \in R^{n}$ such that $u\left(x_{0}\right)=1$;

2) $u$ is fuzzy convex, that is, $u(\lambda x+(1-\lambda) y) \geq \min \{u(x), u(y)\}$

for any $x, y \in R^{n}$ and $0 \leq \lambda \leq 1$;

3) $u$ is upper semicontinuous,

4) $[u]^{0}=c l\left\{x \in R^{n}: u(x)>0\right\}$ is compact.

If $u \in E^{n}$, then $u$ is called a fuzzy number, and $E^{n}$ is said to be a fuzzy number space. For $0<\alpha \leq 1$, denote 


$$
[u]^{\alpha}=\left\{x \in R^{n}: u(x) \geq \alpha\right\} .
$$

Then from (1) - (4), it follows that the $\alpha$-level set $[u]^{\alpha} \in \operatorname{conv}\left(R^{n}\right)$ for all $0 \leq \alpha \leq 1$.

Let $\theta$ be the fuzzy mapping defined by $\theta(x)=0$ if $x \neq 0$ and $\theta(0)=1$.

Define $D: E^{n} \times E^{n} \rightarrow[0, \infty)$ by the relation

$$
D(u, v)=\sup _{0 \leq \alpha \leq 1} h\left([u]^{\alpha},[v]^{\alpha}\right),
$$

where $h$ is the Hausdorff metric defined in $\operatorname{comp}\left(R^{n}\right)$.

Then $D$ is a metric in $E^{n}$.

Further we know that [60]:

1) $\left(E^{n}, D\right)$ is a complete metric space,

2) $D(u+w, v+w)=D(u, v)$ for all $u, v, w \in E^{n}$,

3) $D(\lambda u, \lambda v)=|\lambda| D(u, v)$ for all $u, v \in E^{n}$ and $\lambda \in R$.

Definition 1. [36] A mapping $F:[0, T] \rightarrow E^{n}$ is measurable if for all $\alpha \in[0,1]$ the set-valued map $F_{\alpha}:[0, T] \rightarrow \operatorname{conv}\left(R^{n}\right)$ defined by $F_{\alpha}(t)=[F(t)]^{\alpha}$ is Lebesgue measurable.

Definition 2. [36] A mapping $F:[0, T] \rightarrow E^{n}$ is said to be integrably bounded if there is an integrable function $h(t)$ such that $\|x(t)\| \leq h(t)$ for every $x(t) \in F_{0}(t)$.

Definition 3. [36] The integral of a fuzzy mapping $F:[0, T] \rightarrow E^{n}$ is defined levelwise by

$\left[\int_{0}^{T} F(t) \mathrm{d} t\right]^{\alpha}=\int_{0}^{T} F_{\alpha}(t) \mathrm{d} t$. The set $\int_{0}^{T} F_{\alpha}(t) \mathrm{d} t$ of all

$\int_{0}^{T} f(t) \mathrm{d} t$ such that $f:[0, T] \rightarrow R^{n}$ is a measurable selection for $F_{\alpha}:[0, T] \rightarrow \operatorname{conv}\left(R^{n}\right)$ for all $\alpha \in[0,1]$.

Definition 4. [36] A measurable and integrably bounded mapping $F:[0, T] \rightarrow E^{n}$ is said to be integrable over $[0, T]$ if $\int_{0}^{T} F(t) \mathrm{d} t \in E^{n}$.

Note that if $F:[0, T] \rightarrow E^{n}$ is measurable and integrably bounded, then $F$ is integrable. Further if $F:[0, T] \rightarrow E^{n}$ is continuous, then it is integrable.

Now we consider following control differential equations with the fuzzy parameter

$$
\dot{x}=f(t, x, w, v), x(0)=x_{0},
$$

where $\dot{x}$ means $\frac{\mathrm{d} x}{\mathrm{~d} t} ; t \in R_{+}$is the time; $x \in R^{n}$ is the state; $w \in R^{m}$ is the control; $v \in V \in E^{k}$ is the fuzzy parameter; $f: R_{+} \times R^{n} \times R^{m} \times R^{k} \rightarrow R^{n}$.

Let $W: R_{+} \rightarrow \operatorname{conv}\left(R^{m}\right)$ be the measurable set-valued map.

Definition 5. The set $L W$ of all measurable singlevalued branches of the set-valued map $W(\cdot)$ is the set of the admissible controls.

Further we consider following control fuzzy differen- tial inclusions

$$
\dot{x} \in F(t, x, w), x(0)=x_{0},
$$

where $F: R_{+} \times R^{n} \times R^{m} \rightarrow E^{n}$ is the fuzzy map such that $F(t, x, w) \equiv f(t, x, w, V)$.

Obviously, the control fuzzy differential inclusion (2) turns into the ordinary fuzzy differential inclusion

$$
\dot{x} \in \Phi(t, x), x(0)=x_{0},
$$

if the control $\tilde{w}(\cdot) \in L W$ is fixed and $\Phi(t, x) \equiv F(t, x, \tilde{w}(t))$.

If right-hand side of the fuzzy differential inclusion (3) satisfies some conditions (for example look [12]) then the fuzzy differential inclusions (3) has the fuzzy R-solution.

Let $X(t)$ denotes the fuzzy R-solution of the differential inclusion (3), then $X(t, w)$ denotes the fuzzy R-solution of the control differential inclusion (2) for the fixed $w(\cdot) \in L W$.

Definition 6. The set $Y(T)=\{X(T, w): w(\cdot) \in L W\}$ be called the attainable set of the fuzzy system (2).

\section{The Some Properties of the Fuzzy R-Solution}

Further in the given paper, we consider following control linear fuzzy differential inclusions

$$
\dot{x} \in A(t) x+G(t, w), x(0)=x_{0},
$$

where $A(t)$ is $(n \times n)$ dimensional matrix-valued function; $G: R_{+} \times R^{m} \rightarrow E^{n}$ is the fuzzy map.

In this section, we consider the some properties of the fuzzy R-solution of the control fuzzy differential inclusion (4).

Let the following condition is true.

Condition A:

A1. $A(\cdot)$ is measurable on $[0, T]$;

A2. The norm $\|A(t)\|$ of the matrix $A(t)$ is integrable on $[0, T]$;

A3. The set-valued map $W:\left[t_{0}, T\right] \rightarrow \operatorname{conv}\left(R^{m}\right)$ is measurable on $[0, T]$;

A4. The fuzzy map $G:[0, T] \times R^{m} \rightarrow E^{n}$ satisfies the conditions

1) measurable in $t$;

2) continuous in $w$;

A5. There exist $v(\cdot) \in L_{2}[0, T]$ and $l(\cdot) \in L_{2}[0, T]$ such that

$$
h(W(t), 0) \leq v(t), D(G(t, w), \theta) \leq l(t)
$$

almost everywhere on $[0, T]$ and all $w \in W(t)$.

A6. The set $Q(t)=\{G(t, w(t)): w(\cdot) \in L W\}$ is compact and convex for almost every $t \in[0, T]$, i.e. $Q(t) \in \operatorname{conv}\left(E^{n}\right)$.

Theorem 1. Let the condition A is true. 
Then for every $w(\cdot) \in L W$ there exists the fuzzy $R$-solution $X(\cdot, w)$ such that

1) the fuzzy map $X(\cdot, w)$ has form

$$
X(t, w)=\Phi(t) x_{0}+\Phi(t) \int_{0}^{t} \Phi^{-1}(s) G(s, w(s)) \mathrm{d} s,
$$

where $t \in[0, T] ; \Phi(t)$ is Cauchy matrix of the differential equation $\dot{x}=A(t) x$;

1) $X(t, w) \in E^{n}$ for every $t \in[0, T]$;

2) the fuzzy map $X(\cdot, w)$ is the absolutely continuous fuzzy map on $[0, T]$.

Proof. 1. Show that $X(t, w)$ is the fuzzy R-solution of the fuzzy system (4). We have

$$
\begin{aligned}
& {[X(t, w)]^{\alpha}=\left[\Phi(t) x_{0}+\Phi(t) \int_{0}^{t} \Phi^{-1}(s) G(s, w(s)) \mathrm{d} s\right]^{\alpha}} \\
& =\left[\Phi(t) x_{0}\right]^{\alpha}+\left[\Phi(t) \int_{0}^{t} \Phi^{-1}(s) G(s, w(s)) \mathrm{d} s\right]^{\alpha} \\
& =\Phi(t) x_{0}+\Phi(t) \int_{0}^{t} \Phi^{-1}(s)\left[G(s, w(s)]^{\alpha} \mathrm{d} s\right.
\end{aligned}
$$

for all $\alpha \in[0,1], t \geq 0$ and $w(\cdot) \in L W$. Since $[X(t, w)]^{\alpha}$ is the R-solution of the control differential inclusion

$$
\dot{x} \in A(t) x+\left[G(t, w(t)]^{\alpha}, x\left(t_{0}\right)=x_{0}\right.
$$

(see [30]), we obtain that $X(t, w)$ is fuzzy R-solution of the control fuzzy differential inclusion (4).

2. By [36] and Condition $A$ we have that $X(t, w) \in E^{n}$ for all $t \geq 0$ and $w(\cdot) \in L W$.

3. From [30] we have that $[X(t, w)]^{\alpha}$ is the absolutely continuous set-valued map on $[0, T]$ for all $\alpha \in[0,1]$, i.e. $X(t, w)$ is the absolutely continuous fuzzy map on $[0, T]$. The theorem is proved.

Theorem 2. Let the condition A is true.

Then the attainable set $Y(T)$ is compact and convex.

Proof. It is easy to check that

$$
Y(T)=\Phi(T) x_{0}+\Phi(T) \int_{0}^{T} \Phi^{-1}(s) Q(s) \mathrm{d} s
$$

and

$$
[Y(T)]^{\alpha}=\Phi(T) x_{0}+\int_{0}^{T} \Phi(T) \Phi^{-1}(s)[Q(s)]^{\alpha} \mathrm{d} s,
$$

where $[Q(t)]^{\alpha}:[0, T] \rightarrow \operatorname{conv}\left(\operatorname{conv}\left(R^{n}\right)\right)$ for all $\alpha \in[0,1]$. From $[20,21,30,57]$ we obtain that $\int_{0}^{T} \Phi(T) \Phi^{-1}(s)[Q(s)]^{\alpha} \mathrm{d} s \in \operatorname{conv}\left(\operatorname{conv}\left(R^{n}\right)\right)$ for all $\alpha \in[0,1]$, i.e. $Y(T) \in \operatorname{conv}\left(E^{n}\right)$. This ends the proof. We obtained the basic properties of the fuzzy R-solu- tion of systems (4). Now, we consider the some fuzzy control problems.

\section{Time-Optimal Problems}

Consider the following time-optimal problem: it is necessary to find the minimal time $T$ and the control $w^{*}(\cdot) \in L W$ such that the fuzzy R-solution of system (4) satisfies one of the conditions:

$$
\begin{gathered}
X\left(T, w^{*}\right) \cap S_{k} \neq \varnothing, \\
X\left(T, w^{*}\right) \subset S_{k}, \\
X\left(T, w^{*}\right) \supset S_{k},
\end{gathered}
$$

where $S_{k} \in E^{n}$ is the fuzzy terminal set.

It is obvious that optimum time and optimum controls for these problems will be different.

Theorem 3. (necessary optimal condition for the time-optimal problem (4), (5)). Let the condition $A$ is true and the pair $\left(T, w^{*}(\cdot)\right)$ is optimality of the control problem (4), (5).

Then there exists the vector-function $\psi(\cdot)$, which is the solution of the system

$$
\dot{\psi}=-A^{T}(t) \psi, \psi(T) \in S_{1}(0)
$$

such that

$$
\text { 1) } C\left(\left[G\left(t, w^{*}\right)\right]^{1}, \psi(t)\right)=\max _{w \in W(t)} C\left([G(t, w)]^{1}, \psi(t)\right)
$$

almost everywhere on $[0, T]$;

2) $C\left(\left[X\left(T, w^{*}\right)\right]^{1}, \psi(T)\right)=-C\left(\left[S_{k}\right]^{1},-\psi(T)\right)$,

where $C(P, \psi)=\max _{p \in P}\left(p_{1} \psi_{1}+\cdots+p_{n} \psi_{n}\right), \psi \in R^{n}$, $P \in \operatorname{conv}\left(R^{n}\right)$.

Proof. Let $w^{*}(\cdot)$ is the optimal control and $X\left(\cdot, w^{*}\right)$ is the optimal fuzzy R-solution of the problem (4), (5), i.e.

1) $X\left(T, w^{*}\right) \in Y(T)$;

2) $X\left(T, w^{*}\right) \cap S_{k} \neq \varnothing$.

From 1) and 2) we have

$$
\max _{X \in[Y(T)]^{1}} C(X, \psi) \geq-C\left(\left[S_{k}\right]^{1},-\psi\right)
$$

for all $\psi \in S_{1}(0)$.

Consequently

$$
p=\max _{X \in[Y(T)]^{1}} \min _{\psi \in S_{1}(0)} C(X, \psi)+C\left(\left[S_{k}\right]^{1},-\psi\right) \geq 0 .
$$

From $\left[X\left(T, w^{*}\right)\right]^{1} \cap\left[S_{k}\right]^{1} \neq \varnothing$ we have

$$
q(T, \psi)=C\left(\left[X\left(T, w^{*}\right)\right]^{1}, \psi\right)+C\left(\left[S_{k}\right]^{1},-\psi\right) \geq 0
$$


for all $\psi \in S_{1}(0)$.

From Theorem 1 we have that the function $q(T, \psi)$ is continuous on $R_{+} \times S_{1}(0)$.

If $q(T, \psi)>0$ for all $\psi \in S_{1}(0)$ then we have $q^{0}(T)=\min _{\psi \in S_{1}(0)} q(T, \psi) \geq \gamma>0$. Hence there exists $\tau<T$ such that $q^{0}(\tau) \geq 0$. Consequently we have

$$
C\left(\left[X\left(\tau, w^{*}\right)\right]^{1}, \psi\right)+C\left(\left[S_{k}\right]^{1},-\psi\right) \geq 0
$$

for all $\psi \in S_{1}(0)$, i.e. $\left[X\left(\tau, w^{*}\right)\right]^{1} \cap\left[S_{k}\right]^{1} \neq \varnothing$.

It contradicts that $T$ is optimal time.

If, $p>0$

$$
\begin{aligned}
& \max _{X \in[Y(T)]} \min _{\psi \in S_{1}(0)} C(X, \psi)+C\left(\left[S_{k}\right]^{1},-\psi\right) \\
& =C(\tilde{X}, \tilde{\psi})+C\left(\left[S_{k}\right]^{1},-\tilde{\psi}\right)
\end{aligned}
$$

and $\left[X\left(T, w^{*}\right)\right]^{1} \neq \tilde{X}$, than we have a contradiction. Hence there exists $\tilde{\psi} \in S_{1}(0)$ such that

$$
\begin{gathered}
C\left(\left[X\left(T, w^{*}\right)\right]^{1}, \tilde{\psi}\right)=\max _{X \in[Y(T)]^{1}} C(X, \tilde{\psi}), \\
C\left(\left[X\left(T, w^{*}\right)\right]^{1}, \tilde{\psi}\right)=-C\left(\left[S_{k}\right]^{1},-\tilde{\psi}\right) .
\end{gathered}
$$

Consequently

$$
\begin{aligned}
& C\left(\int_{0}^{T} \Phi(T) \Phi^{-1}(s)\left[G\left(s, w^{*}(s)\right)\right]^{1} \mathrm{~d} s, \tilde{\psi}\right) \\
& =\max _{w(\cdot) \in L W} C\left(\int_{0}^{T} \Phi(T) \Phi^{-1}(s)[G(s, w(s))]^{1} \mathrm{~d} s, \tilde{\psi}\right)
\end{aligned}
$$

Then we have

$$
\begin{aligned}
& C\left(\Phi(T) \Phi^{-1}(s)\left[G\left(s, w^{*}(s)\right)\right]^{1}, \tilde{\psi}\right) \\
& =\max _{w \in W(t)} C\left(\Phi(T) \Phi^{-1}(s)[G(s, w)]^{1}, \tilde{\psi}\right)
\end{aligned}
$$

for almost every $s \in[0, T]$. If

$$
\psi(t)=\frac{\left(\Phi(T) \Phi^{-1}(t)\right)^{T} \tilde{\psi}}{\left\|\left(\Phi(T) \Phi^{-1}(t)\right)^{T} \tilde{\psi}\right\|},
$$

than the theorem 3 is proved.

Theorem 4. (necessary optimal condition for the time-optimal problem (4), (6)). Let the condition $A$ is true and the pair $\left(T, w^{*}(\cdot)\right)$ is optimality of the control problem (4), (6).

Then there exist the vector-function $\psi(\cdot)$, which is the solution of the system

$$
\dot{\psi}=-A^{T}(t) \psi, \psi((T)) \in S_{1}(0)
$$

and $\beta \in[0,1]$ such that

1)

$$
C\left(\left[G\left(t, w^{*}\right)\right]^{\beta}, \psi(t)\right)=\max _{w \in W(t)} C\left([G(t, w)]^{\beta}, \psi(t)\right)
$$

almost everywhere on $[0, T]$;

2) for all $\alpha \in[0,1]$

$$
C\left(\left[X\left(T, w^{*}\right)\right]^{\alpha}, \psi(T)\right) \leq C\left(\left[S_{k}\right]^{\alpha}, \psi(T)\right)
$$

and

$$
C\left(\left[X\left(T, w^{*}\right)\right]^{\beta}, \psi(T)\right)=C\left(\left[S_{k}\right]^{\beta}, \psi(T)\right) .
$$

This theorem is proved analogous theorem 5 with little changes of condition (8):

for all $\alpha \in[0,1]$

$$
\max _{X \in[Y(T)]^{\alpha}} C(X, \psi)-C\left(\left[S_{k}\right]^{\alpha}, \psi\right) \leq 0
$$

for all $\psi \in S_{1}(0)$ and there exist $\beta \in[0,1]$ and $\bar{\psi} \in R^{n}$ such that

$$
\max _{X \in[Y(T)]^{\beta}} C(X, \bar{\psi})-C\left(\left[S_{k}\right]^{\beta}, \bar{\psi}\right)=0 .
$$

Theorem 5. (necessary optimal condition for the time-optimal problem (4), (7)). Let the condition $A$ is true and the pair $\left(T, w^{*}(\cdot)\right)$ is optimality of the control problem (4), (7).

Then there exist the vector-function $\psi(\cdot)$, which is the solution of the system

$$
\dot{\psi}=-A^{T}(t) \psi, \psi(T) \in S_{1}(0)
$$

and $\beta \in[0,1]$ such that

1)

$$
C\left(\left[G\left(t, w^{*}\right)\right]^{\beta}, \psi(t)\right)=\max _{w \in W(t)} C\left([G(t, w)]^{\beta}, \psi(t)\right)
$$

almost everywhere on $[0, T]$;

2) for all $\alpha \in[0,1]$

$$
C\left(\left[X\left(T, w^{*}\right)\right]^{\alpha}, \psi(T)\right) \geq C\left(\left[S_{k}\right]^{\alpha}, \psi(T)\right)
$$

and

$$
C\left(\left[X\left(T, w^{*}\right)\right]^{\beta}, \psi(T)\right)=C\left(\left[S_{k}\right]^{\beta}, \psi(T)\right) .
$$

Also this theorem is proved analogous theorem 5 with little changes of condition (8):

for all $\alpha \in[0,1]$

$$
\max _{X \in[Y(T)]^{\alpha}} C(X, \psi)-C\left(\left[S_{k}\right]^{\alpha}, \psi\right) \geq 0,
$$

for all $\psi \in S_{1}(0)$ and there exist $\beta \in[0,1]$ and $\bar{\psi} \in R^{n}$ such that 


$$
\max _{X \in[Y(T)]^{\beta}} C(X, \bar{\psi})-C\left(\left[S_{k}\right]^{\beta}, \bar{\psi}\right)=0 .
$$

Example. Consider the following control linear fuzzy differential inclusions

$$
\dot{x} \in x+w+F, x(0)=0,
$$

where $x$ is the state; $w \in W=[-1,1]$ is the control; $F \in E^{1}$ is the fuzzy set, where

$$
v(f)=\left\{\begin{array}{cc}
0, & f \leq 0,5 \\
2 f-1, & 0,5<f<1 \\
-2 f+3, & 1 \leq f<1,5 \\
0, & f>1,5
\end{array} .\right.
$$

Consider the following time-optimal problem: it is necessary to find the minimal time $T$ and the control $w^{*}(\cdot) \in L W$ such that the fuzzy R-solution of system (9) satisfies of the condition (5), where the fuzzy terminal set $S_{k} \in E^{1}$ such, that

$$
\sigma(x)=\left\{\begin{array}{cc}
0, & x<1,75 \\
4 x-7, & 1,75 \leq x<2 \\
1, & 2 \leq x<3 \\
-4 x+13, & 3 \leq x<3,25 \\
0, & 3,25<x
\end{array} .\right.
$$

The control $w^{*}(t)=1$ and time $T=\ln (2)$ will be optimum pair for the given problem. Fuzzy set $X\left(T, w^{*}\right)$ and fuzzy terminal set $S_{K}$ are shown in Figure 1.

Obviously, this optimal pair satisfies to conditions of the theorem 3:

1) $\left(w^{*}(t), \psi(t)\right)=C(W, \psi(t))$ for almost every $t \in[0, \ln (2)]$;

2) $C\left(\left[X\left(T, w^{*}\right)\right]^{1}, \psi(T)\right)=-C\left(\left[S_{k}\right]^{1},-\psi(T)\right)$, where $\psi(t)=1$ for almost every $t \in[0, \ln (2)]$, $\left[X\left(T, w^{*}\right)\right]^{1}=2, \cdot\left[S_{K}\right]^{1}=[2,3]$

If we consider the time-optimal problem (9), (6) then the control $w^{*}(t)=1$ and time $T=\ln \left(\frac{13}{6}\right)$ will be optimum pair. Fuzzy set $X\left(T, w^{*}\right)$ and fuzzy terminal set $S_{K}$ are shown in Figure 2. This optimal pair satisfies to conditions of the theorem 4 :

1) $\left(w^{*}(t), \psi(t)\right)=C(W, \psi(t))$ for almost every $t \in\left[0, \ln \left(\frac{13}{6}\right)\right]$;

2) $C\left(\left[X\left(T, w^{*}\right)\right]^{0},-\psi(T)\right)=C\left(\left[S_{k}\right]^{0},-\psi(T)\right)$,

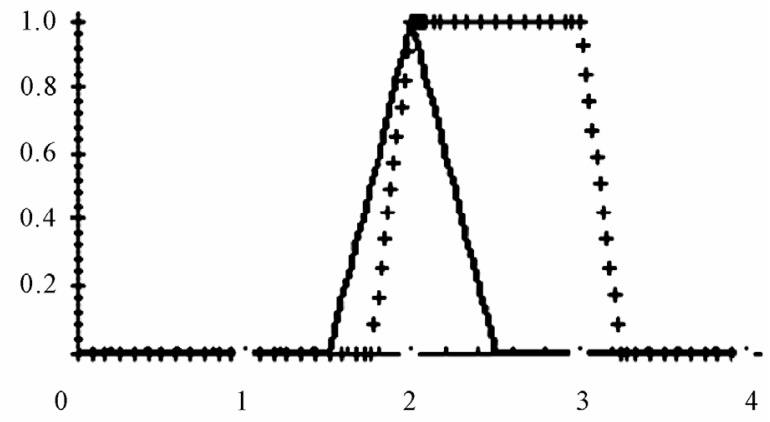

Figure 1. $X(\ln (2), 1)(-), S_{K}(+)$.

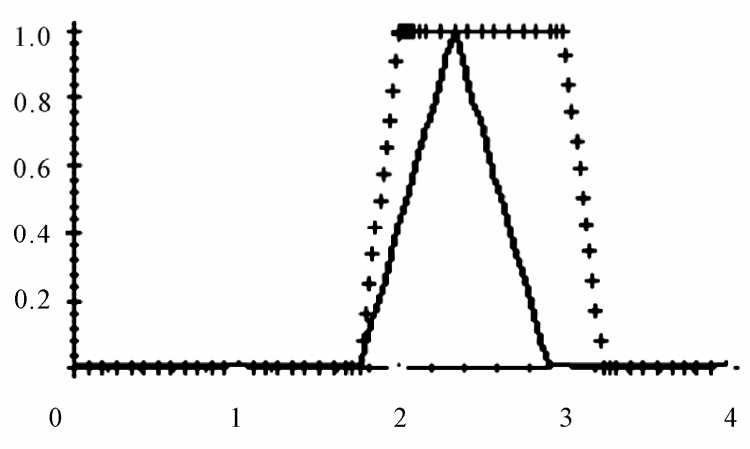

Figure 2. $X\left(\ln \left(\frac{13}{6}\right), 1\right)(-), S_{K}{ }^{(+)}$.

where $\psi(t)=1$ for almost every $t \in\left[0, \ln \left(\frac{13}{6}\right)\right]$,

$\left[X\left(T, w^{*}\right)\right]^{0}=\left[\frac{7}{4}, \frac{35}{12}\right],\left[S_{K}\right]^{0}=\left[\frac{7}{4}, \frac{13}{4}\right]$.

If we consider a problem (9), (7) it is obvious that the solution does not exist.

\section{Conclusions}

In the last decades, a number of works devoted to problems of optimal control of set-valued trajectories (fuzzy trajectories, trajectory bundles or an ensemble of trajectories) appeared; these works fall into a subdivision of the optimal control theory, namely, the theory of process control under uncertainty and fuzzy conditions. This is conditioned by the fact that, in actual problems arising in economy and engineering in the course of construction of a mathematical model, it is practically impossible to exactly describe the behavior of an object. This is explained by the following fact. First, for some parameters of the object, it impossible to specify exact values and laws of their change, but it is possible to determine the domain of these changes. Second, for the sake of simplicity of the mathematical model being constructed, the 
equations that describe the behavior of the object are simplified and one should estimate the consequences of such a simplification. Therefore, if is possible to divide the articles devoted to this direction into two types characterized by the following distinctive features:

1) there exists an incomplete or fuzzy information on the initial data;

2) the equations describing the behavior of the object to be controlled are assumed to be inexact, for example, they can contain some parameters whose exact values and laws of variation are unknown but the domain of their values is fuzzy.

In the second case, fuzzy differential inclusions are frequently used to describe behavior of objects. The reason is that, first this approach is most obvious and, second, theory of fuzzy and ordinary differential inclusions is well found and is rapidly developed at the present time.

In the present paper, the necessary conditions of optimal of control for a system of the latter form of equations with the fuzzy R-solutions are formulated and proved.

\section{References}

[1] A. Marchaud, "Sur les Champs de Demicones et Equations Differentielles du Premier Order," Bulletin de la Société Mathématique de France, Vol. 62, 1934, pp. 1-38.

[2] A. Marchaund, "Sur les Champs des Deme-Droites et les Equations Differentilles du Premier Ordre,” Bulletin de la Société Mathématique de France, Vol. 63, 1934, pp. 1-38.

[3] S.C. Zaremba, "Sur une Extension de la Notion d'Equation Differentielle," Reports of the Paris Academy of Sciences, Vol. 199, 1934, pp. 1278-1280.

[4] T. Wazewski, "Systemes de Commande et Equations au Contingent,” Bulletin de l'Academie Polonaise des Sciences. Serie des Sciences Mathematiques, Astronomiques et Physiques, No. 9, 1961, pp. 151-155.

[5] T. Wazewski, "Sur une Condition Equivalente e L'Equation au Contingent," Bulletin de l'Academie Polonaise des Sciences. Serie des Sciences Mathematiques, Astronomiques et Physiques, No. 9, 1961, pp. 865-867.

[6] A. F. Filippov, "Classical Solutions of Differential Equations with Multi-Valued Right-Hand Side,” SIAM Journal on Control and Optimization, Vol. 5, No. 4, 1967, pp. 609-621. doi:10.1137/0305040

[7] J.-P. Aubin and A. Cellina, "Differential Inclusions. Set-Valued Maps and Viability Theory.” Springer-Verlag, Berlin-Heidelberg-New York-Tokyo, 1984.

[8] V. A. Plotnikov, A. V. Plotnikov and A. N. Vityuk, "Differential Equations with Multivalued Right-Hand Sides,” Asymptotics Methods, AstroPrint, Odessa, 1999.

[9] G. V. Smirnov, "Introduction to the Theory of Differential Inclusions,” Graduate Studies in Mathematics, Vol.
41, American Mathematical Society, Providence, 2002.

[10] J.-P. Aubin, "Mutational Equations in Metric Spaces," Set-Valued Analysis, Vol. 1, No. 1, 1993, pp. 3-46. doi:10.1007/BF01039289

[11] J.-P. Aubin and H. Frankovska, "Set-Valued Analysis," Birkhauser, Systems and Control: Fundations and Applications, 1990.

[12] A. I. Panasyuk, "Quasidifferential Equations in a Metric Space,” Differentsial'nye Uravneniya, Vol. 21, No. 8, 1985, pp. 1344-1353.

[13] A. I. Panasyuk and V. I. Panasyuk, "Asymptotic Turnpike Optimization of Control Systems," Nauka i Tekhnika, Minsk, 1986.

[14] A. A. Tolstonogov, "On an Equation of an Integral Funnel of a Differential Inclusion,” Matematicheskie Zametki, Vol. 32, No. 6, 1982, pp. 841-852.

[15] D. A. Ovsyannikov, "Mathematical Methods for the Control of Beams,” Leningrad University, Leningrad, 1980.

[16] V. I. Zubov, "Dynamics of Controlled Systems,” Vyssh Shkola, Moscow, 1982.

[17] V. I. Zubov, "Stability of Motion. Lyapunov Methods and Their Application,” Vyssh Shkola, Moscow, 1984.

[18] A. V. Arsirii and A. V. Plotnikov, "Systems of Control over Set-Valued Trajectories with Terminal Quality Criterion," Ukrainian Mathematical Journal, Vol. 61, No. 8, 2009, pp. 1349-1356. doi:10.1007/s11253-010-0280-3

[19] N. D. Phu and T. T. Tung, "Some Results on Sheaf-Solutions of Sheaf Set Control Problems," Nonlinear Analysis, Vol. 67, No. 5, 2007, pp. 1309-1315. doi:10.1016/j.na.2006.07.018

[20] A. V. Plotnikov, "Controlled Quasidifferential Equations and Some of Their Properties," Differential Equations, Vol. 34, No. 10, 1998, pp. 1332-1336.

[21] V. A. Plotnikov and A. V. Plotnikov, "Multivalued Differential Equations and Optimal Control," Applications of Mathematics in Engineering and Economics, Heron Press, Sofia, 2001, pp. 60-67.

[22] G. N. Konstantinov, "Sufficient Conditions for Optimality of a Minimax Control Problem of an Ensemble of Trajectories," Soviet Doklady Mathematics, Vol. 36, No. 3, 1988, pp. 460-463.

[23] S. Otakulov, "On the Approximation of the Time-Optimality Problem for Controlled Differential Inclusions," Cybernetics and Systems Analysis, Vol. 30, No. 3, 1994, pp. 458-462. doi:10.1007/BF02366480

[24] S. Otakulov, "On a Difference Approximation of a Control System with Delay,” Automation and Remote Control, Vol. 69, No. 4, 2008, pp. 690-699. doi:10.1134/S0005117908040152

[25] A. V. Plotnikov, "Linear Control Systems with Multivalued Trajectories,” Kibernetika, No. 4, 1987, pp. 130131.

[26] A. V. Plotnikov, "Compactness of the Attainability Set of a Nonlinear Differential Inclusion that Contains a Control,” Kibernetika, No. 6, 1990, pp. 116-118.

[27] A. V. Plotnikov, "A Problem on the Control of Pencils of 
Trajectories,” Siberian Mathematical Journal, Vol. 33, No. 2, 1992, pp. 351-354. doi:10.1007/BF00971112

[28] A. V. Plotnikov, "Two Control Problems under Uncertainty Conditions," Cybernetics and Systems Analysis, Vol. 29, No. 4, 1993, pp. 567-573. doi:10.1007/BF01125871

[29] A. V. Plotnikov, "Necessary Optimality Conditions for a Nonlinear Problems of Control of Trajectory Bundles," Cybernetics and Systems Analysis, Vol. 36, No. 5, 2000, pp. 729-733. doi:10.1023/A:1009432907531

[30] A. V. Plotnikov, "Linear Problems of Optimal Control of Multiple-Valued Trajectories," Cybernetics and Systems Analysis, Vol. 38, No. 5, 2002, pp. 772-782. doi:10.1023/A:1021899111846

[31] A. V. Plotnikov and T. A. Komleva, "Some Properties of Trajectory Bunches of a Controlled Bilinear Inclusion," Ukrainian Mathematical Journal, Vol. 56, No. 4, 2004, pp. 586-600. doi:10.1007/s11253-005-0114-X

[32] A. V. Plotnikov and L. I. Plotnikova, "Two Problems of Encounter under Conditions of Uncertainty,” Journal of Applied Mathematics and Mechanics, Vol. 55, No. 5, 1991, pp. 618-625. doi:10.1016/0021-8928(91)90108-7

[33] L. A. Zadeh, "Fuzzy Sets," Information and Control, No. 8, 1965, pp. 338-353. doi:10.1016/S0019-9958(65)90241-X

[34] B. Bede and S. G. Gal, "Solutions of Fuzzy Differential Equations Based on Generalized Differentiability," Communications in Mathematical Analysis, Vol. 9, No. 2, 2010, pp. 22-41.

[35] M. H. Chen, D. H. Li and X. P. Xue, "Periodic Problems of First Order Uncertain Dynamical Systems,” Fuzzy Sets and Systems, Vol. 162, No. 1, 2011, pp. 67-78. doi:10.1016/j.fss.2010.09.011

[36] O. Kaleva, “Fuzzy Differential Equations,” Fuzzy Sets and Systems, Vol. 24, No. 3, 1987, pp. 301-317. doi:10.1016/0165-0114(87)90029-7

[37] O. Kaleva, "A Note on Fuzzy Differential Equations," Nonlinear Analysis, Vol. 64, No. 5, 2006, pp. 895-900. doi:10.1016/j.na.2005.01.003

[38] T. A. Komleva, "The Full Averaging of Linear Fuzzy Differential Equations with 2pi-Periodic Right-Hand Side," Journal of Advanced Research in Dynamical and Control Systems, Institute of Advanced Scientific Research, USA, Vol. 3, No. 1, 2011, pp. 12-25.

[39] T. A. Komleva, A. V. Plotnikov and N. V. Skripnik, "Differential Equations with Set-Valued Solutions," Ukrainian Mathematical Journal, Vol. 60, No. 10, 2008, pp. 1540-1556. doi:10.1007/s11253-009-0150-z

[40] V. Lakshmikantham, T. Gnana Bhaskar and D. J Vasundhara, "Theory of Set Differential Equations in Metric Spaces,” Cambridge Scientific Publishers, Cambridge, 2006.

[41] V. Lakshmikantham and R. N. Mohapatra, "Theory of Fuzzy Differential Equations and Inclusions,” Series in Mathematical Analysis and Applications, Vol. 6, Taylor \& Francis, Ltd., London, 2003.
[42] J. Y. Park and H. K. Han, "Existence and Uniqueness Theorem for a Solution of Fuzzy Differential Equations," International Journal of Mathematics and Mathematical Sciences, Vol. 22, No. 2, 1999, pp. 271-279. doi:10.1155/S0161171299222715

[43] J. Y. Park and H. K. Han, "Fuzzy Differential Equations," Fuzzy Sets and Systems, Vol. 110, No. 1, 2000, pp. 69-77. doi:10.1016/S0165-0114(98)00150-X

[44] A. V. Plotnikov and T. A. Komleva, "The Full Averaging of Linear Fuzzy Differential Equations,” Journal of Advanced Research in Differential Equations, Institute of Advanced Scientific Research, USA, Vol. 2, No. 3, 2010, pp. 21-34.

[45] A. V. Plotnikov and N. V. Skripnik, "Differential Equations with 'Clear' and Fuzzy Multivalued Right-Hand Sides,” Asymptotics Methods, AstroPrint, Odessa, 2009.

[46] S. Seikkala, “On the Fuzzy Initial Value Problem,” Fuzzy Sets and Systems, Vol. 24, No. 3, 1987, pp. 319-330. doi:10.1016/0165-0114(87)90030-3

[47] D. Vorobiev and S. Seikkala, "Towards the Theory of Fuzzy Differential Equations,” Fuzzy Sets and Systems, Vol. 125, No. 2, 2002, pp. 231-237. doi:10.1016/S0165-0114(00)00131-7

[48] J.-P. Aubin, "Fuzzy Differential Inclusions," Problems of Control and Information Theory, Vol. 19, No. 1, 1990, pp. 55-67.

[49] V. A. Baidosov, "Differential Inclusions with Fuzzy Right-Hand Side,” Soviet Mathematics, Vol. 40, No. 3, 1990, pp. 567-569.

[50] V. A. Baidosov, "Fuzzy Differential Inclusions,” Journal of Applied Mathematics and Mechanics, Vol. 54, No. 1, 1990, pp. 8-13. doi:10.1016/0021-8928(90)90080-T

[51] E. Hullermeier, "An Approach to Modelling and Simulation of Uncertain Dynamical Systems," International Journal of Uncertain, Fuzziness Knowledge-Based Systems, Vol. 5, No. 2, 1997, pp. 117-137. doi:10.1142/S0218488597000117

[52] A. V. Plotnikov, T. A. Komleva and L. I. Plotnikova, "The Partial Averaging of Differential Inclusions with Fuzzy Right-Hand Side,” Journal Advanced Research in Dynamical \& Control Systems, Institute of Advanced Scientific Research, USA, Vol. 2, No. 2, 2010, pp. 26-34.

[53] A. V. Plotnikov, T. A. Komleva and L. I. Plotnikova, “On the Averaging of Differential Inclusions with Fuzzy Right-Hand Side When the Average of the Right-Hand Side Is Absent," Iranian Journal of Optimization, Vol. 2, No. 3, 2010, pp. 506-517.

[54] T. E. Dabbous, "Adaptive Control of Nonlinear Systems Using Fuzzy Systems,” Journal of Industrial and Management Optimization, Vol. 6, No. 4, 2010, pp. 861-880. doi:10.3934/jimo.2010.6.861

[55] A. V. Plotnikov and T. A. Komleva, "Linear Problems of Optimal Control of Fuzzy Maps,” Intelligent Information Management, Vol. 1, No. 3, 2009, pp. 139-144. doi:10.4236/iim.2009.13020

[56] A. V. Plotnikov, T. A. Komleva and A. V. Arsiry, "Necessary and Sufficient Optimality Conditions for a Control 
Fuzzy Linear Problem,” Internatioal Journal of Industrial Mathematics, Vol. 1, No. 3, 2009, pp. 197-207.

[57] A. V. Plotnikov and T. A. Komleva, "Fuzzy Quasidifferential Equations in Connection with the Control Problems," International Journal of Open Problems in Computer Science and Mathematics, Vol. 3, No. 4, 2010, pp. 439-454.

[58] I. V. Molchanyuk and A. V. Plotnikov, "Linear Control Systems with a Fuzzy Parameter," Nonlinear Oscillator, Vol. 9, No. 1, 2006, pp. 59-64.

doi:10.1007/s11072-006-0025-2
[59] A. V. Plotnikov, T. A. Komleva and I. V. Molchanyuk, "Linear Control Problems of the Fuzzy Maps," Journal of Software Engineering \& Applications, Scientific Research Publishing, Inc., USA, Vol. 3, No. 3, 2010, pp. 191-197. doi:10.4236/jsea.2010.33024

[60] M. L. Puri and D. A. Ralescu, "Fuzzy Random Variables," Journal of Mathematical Analysis and Applications, No. 114, 1986, pp. 409-422. doi:10.1016/0022-247X(86)90093-4 